[RADIOCARbon, Vol. 8, 1966, P. 239-247]

\title{
KÖLN RADIOCARBON MEASUREMENTS I
}

\section{H. SCHWABEDISSEN and J. FREUNDLICH \\ University of Cologne,* Germany}

\section{INTRODUCTION}

After publication of the occurrence of $\mathrm{C}^{14}$ in nature and the first successful datings made by the radiocarbon method (Anderson et al., 1947; Arnold and Libby, 1949), a fair number of physicists as well as geologists, paleobotanists, and prehistorians in Germany became interested (e.g., Harteck, 1951; Zeuner, 1951; Firbas, 1953; Schwabedissen, 1949) in studying the applicability of the new method. So, one of us (H.S.) together with F. Firbas tried to stimulate plans by F. Houtermans (later at Bern, Switzerland) to organize a dating laboratory at Göttingen, before 1950. When the Heidelberg laboratory was founded, close contact was kept with O. Haxel and K. O. Münnich by providing them with carefully selected samples covering a variety of prehistoric ages (partly published in Heidelberg I). Samples were also submitted to Washington, D. C. (see USGS III, I, II), Yale (see Yale III), and Copenhagen (unpub.).

Plans for our laboratory were initiated after a meeting of $\mathrm{K}$. Beckhoff** and Schwabedissen at a prehistoric conference held at Münster/ W. in April, 1957. During 1957 through 1959, Beckhoff, an electronic engineer and owner of an electroengineering plant, designed and built the equipment (Beckhoff, 1960) which, after showing satisfactory performance, was transferred to Cologne in December, 1959. At the same time, H. Kirchner assumed responsibility for the new-founded $\mathrm{C}^{14}$ laboratory; from summer 1960 until he left in summer 1962, he began to build up a complete second counting system of similar design.

From January, 1962, the electronic parts of the Beckhoff equipment have been working dependably. In January, 1963, Freundlich assumed responsibilty for the $\mathrm{C}^{14}$ laboratory, and was able to undertake routine dating operations by the middle of 1963. In May, 1964, K. Beckhoff's equipment was given as a donation to the University of Cologne. The equipment devised by $\mathrm{H}$. Kirchner has been taken care of by $\mathrm{H} . \mathrm{H}$. Eipper from March, 1963; it is intended to begin the dating routine in the near future. A third system is presently being established on funds from the Volkswagenwerkstiftung, mainly in order to date samples of higher ages.

\section{METHODS}

After careful manual selection, the sample is boiled with $5 \% \mathrm{HCl}$, and then with $10 \% \mathrm{NaOH}$ solution followed again by $5 \% \mathrm{HCl}$; between these steps, the sample is washed with boiling distilled water to the

*Institut für Ur- und Frühgeschichte der Universität zu Köln. Germany.

** Bodendenkmalpfleger (= member of archaeological survey), Witten/Ruhr, 
neutral point and subsequently dried and weighed, unless stated otherwise in the individual sample description. The purification of the counter-filling gas $\left(\mathrm{CO}_{2}\right.$, gained by controlled ignition similar to De Vries' device, see De Vries and Barendsen, 1953) is done in the "wet" way as described by Haxel (1957, p. 166); for the decomposition of the $\mathrm{CaCO}_{3}$, approx. $2 \mathrm{~N} \mathrm{HCl}$ is applied (Calvin et al., 1960, p. 153ff.). The resulting $\mathrm{CO}_{2}$ then passes high efficiency drying columns held at $-80^{\circ} \mathrm{C}$ and is stored in $6 \mathrm{~L}$ bulbs for subsequent measurement. As a control of the $\mathrm{CO}_{2}$ purity, the plateau rise is routinely determined before and after each single measurement. The purity of the $\mathrm{CO}_{2}$ gained normally proved to be excellent; in rare cases, when the plateau rise seemed to be detectably "shifted" towards higher counter voltage value (i.e., more than 10 to $20 \mathrm{v}$ ), the performance could reliably be improved by passing the gas over hot silver. The electronic amplification, too, is checked at regular intervals.

The $\mathrm{CO}_{2}$ is filled to a pressure of $710 \mathrm{~mm} \mathrm{Hg}$ (at $23^{\circ} \mathrm{C}$ ) into the 2-L copper-walled proportional counter (of Heidelberg type). The counter is shielded by $10 \mathrm{~cm}$ of selected old lead and by an anti-coincidence shield of 15 commercial Geiger counters of the cosmic-ray type (model HZ-100, Zentralwerkstatt Göttingen). The anti-coincidence background count is $8.5 \mathrm{cpm}$ and is averaged over a "floating" period of 20 days. A barometric effect of $0.08+0.024 \mathrm{cpm}$ per $\mathrm{cm} \mathrm{Hg}$ was measured during October, 1964, when extreme variations of the atmospheric pressure occurred. In conjunction with a low but reproducibly time-dependent increase of the alpha-count rate found for any $\mathrm{CO}_{2}$-filling which is allowed to stay in the counter tube for several days, a concurrent increase of the net beta-count was observed, the ratio of increase, $\Delta \beta / \Delta \alpha$, being close to three (Freundlich, 1965). The net contemporary value $(95 \%$ NBS oxalic acid) is $11.8 \mathrm{cpm}$. As a control of the statistical regularity of the background and beta counts, the time elapsing between each 100 anti-coincidence counts is printed out. Each dating gas is measured for at least three 16-hr periods at two or more independent fillings more than 14 days apart.

The dates are based on Libby's half life value, $5570 \mathrm{yr}$; the standard deviations given with the $\mathrm{C}^{14}$ ages are rounded-off values calculated from the statistical uncertainties of the count rates of sample, background, and contemporary standard; a minimum standard deviation of $80 \mathrm{yr}$ is included to take account of the De Vries effect.

From the 50 radiocarbon dates determined so far in this laboratory (all of them by means of the Beckhoff apparatus), only those have been included in this list which can either be checked with radiocarbon dates of other laboratories made on material of exactly the same sample (section II), or those which can be compared with age determinations independently based on historical, archaeological, or geological reasons (section I). 
ACKNOWLEDGMENTS

The authors would like to express their thanks to O. Haxel and K. O. Münnich for their helpful collaboration. Thanks are also due to Max Rutloh, for all his assistance given with the construction of the dating apparatus, and to Miss E. Spiess for excellent laboratory work with the sample preparation.

\section{SAMPLE DESCRIPTIONS}

I. GEOLOGIC AND ARCHAEOLOGIC SAMPLES

\section{KN-53. Querenstede}

$11,070 \pm 320$

9120 B.c.

Wood charcoal found in Alleröd layer, $1.30 \mathrm{~m}$ below surface of Circular-Ditch Graves (Kreisgraben-Gräberfeld) site, Querenstede, Gemeinde Zwischenahn, Landkreis Ammerland, Niedersachsen $\left(53^{\circ} 11^{\prime} \mathrm{N}\right.$ Lat, $7^{\circ} 00^{\prime}$ E Long), Germany. Geological classification: Alleröd, by A. Bohmers and H. T. Waterbolk (private commun.). Excavated 1961 and subm. by D. Zoller, Staatliches Mus. für Vorgeschichte, Oldenburg (Oldb.), Niedersachsen (see Zoller, 1963). Comment: numerous $\mathrm{C}^{14}$ dates from Alleröd period have been discussed; they lie consistently between 8800 and 10,000 B.c. (e.g., Gross, 1952, p. 74; 1954, p. 192; 1955, p. 110), in accordance with the Swedish varve chronology.

\section{KN-72. Los Millares}

$4380 \pm 120$

Wood charcoal of mixed composition (det. Mrs. Maura Scannell, Natl. Mus. of Antiquities, Dublin), coll. 1956 by A. Arribas from Grave no. 19, during excavation campaign by M. Almagro, Inst. Espanol de Prehistoria, Madrid, and A. Arribas, Museo Arqueologico, Barcelona; of well-known site of Los Millares near Santa Fé de Mondújar, prov. Almeria (36 $59^{\prime} \mathrm{N}$ Lat, $2^{\circ} 28^{\prime} \mathrm{W}$ Long), Spain (Almagro and Arribas, 1963), a place already excavated in 1892 by L. Siret. Sample (by commission of H. Schubart, Deutsches Archäologisches Institut, Madrid) subm. by E. Sangmeister, Univ. of Freiburg/Breisgau. Comment: archaeological age was determined (Sangmeister and Do Paço, 1956, p. 229) to be ca. 2400 B.c.; material from same site (sample Sch-45) was dated by Heidelberg lab.: H-204/247, Los Millares, $2345 \pm 85$ B.c. (see Schwabedissen and Münnich, 1958).

\section{KN-90. Bogazköy}

$3490 \pm 120$

Large sample of wood charcoal from ceiling of a burnt building belonging to castle of Büyükkale, citadel of the ancient Hethitian city at Bogazköy (modern name Bogazkale), villayet Corum (40 02' N Lat, $34^{\circ}$ $33^{\prime}$ E Long), Turkey. Taken from $1.20 \mathrm{~m}$ below surface, during excavations of the 1959 Bogazköy expedition of German Archaeol. Inst.; subm. by K. Bittel, Deutsches Archäologisches Institut, Berlin (see Bittel et al., 
1957). Comment: archaeological age, corresponding to Old Assyrian trade settlements in Cappadocia: early second millennium B.c. (K. Bittel).

\section{KN-7. Asberg}

$1990 \pm 80$

40 B.c.

Charred cereal grains from excavations of Roman military camp Asciburgium (=Asberg), Kreis Moers, Nordrhein-Westfalen $\left(51^{\circ} 25^{\prime} \mathrm{N}\right.$ Lat, $6^{\circ} 40^{\prime} \mathrm{E}$ Long), Germany. Position: Trench IV, no. 19 P $54+30$. Coll. 1961 and subm. 1963 by F. Tischler, Niederrheinisches Mus., Duisburg. Sample has only been subjected to acid pretreatment. Comment: sample found in Claudian layer, age must be close to A.D. 50 (F. Tischler, to be published).

\section{KN-3. Neuss}

$$
1900 \pm 120
$$

Wood charcoal with remains of cremation from incineration hole, Place no. 1202, from excavations of Roman military camp Novaesium (see Petrikovits, 1957), close to modern town of Neuss, NordrheinWestfalen ( $51^{\circ} 12^{\prime} \mathrm{N}$ Lat, $6^{\circ} 41^{\prime} \mathrm{E}$ Long), Germany. Sample coll. 1958; present leader of excavations, G. Müller, Rheinisches Landesmuseum, Aussenstelle Neuss. Subm. 1959, by Ph. Filtzinger, now Württembergisches Landesmuseum, Stuttgart. Comment: archaeological age depending on finds of ceramics (terra sigillata) within the hole: final first century A.D. (Mrs. M. Vegas, Rheinisches Landesmuseum, Bonn, and G. Müller; G. Müller, to be published).

\section{KN-50. Oberaden}

$2050 \pm 120$

Wood (Picea, det. Miss I. Peters, Bonn) from the timbering of a barrel-type water well (=Fassbrunnen), Well no. 1, outside the camp area of Roman military camp of Oberaden, Kreis Unna, NordrheinWestfalen (51 $36^{\prime} \mathrm{N}$ Lat, $7^{\circ} 37^{\prime}$ E Long), Germany. Sample coll. and subm. by H. Beck, Landesmuseum für Vor- und Frühgeschichte, Münster/W. (Aschemeyer, 1963; see also Albrecht, 1938/1942). Comment: historical date: exactly, 11 to 3 B.c. It is supposed that no spruce trees (Picea) were growing at that time within at least $100 \mathrm{~km}$, so that timber must have been brought from a fair distance (R. Schütrumpf).

KN-40. Peiting

\section{A.D. 1110}

$840 \pm 80$

Wood charcoal (Pinus) from cover of a coffin containing a female corpse found $50 \mathrm{~cm}$ below surface in "Schwarzer Laich" bog near Peiting ( $47^{\circ} 47^{\prime} \mathrm{N}$ Lat, $10^{\circ} 55^{\prime} \mathrm{E}$ Long), close to Schongau, Bayern, Germany. Coll. 1957 and subm. by K. Schlabow, Textilmuseum, Neumünster. Comment: from the clothes and textiles, the find can be attributed to Earlv Middle Ages (see Schlabow, 1961). 


\section{KN-35. Berlin Nr. 45}

$$
640 \pm 80
$$

Wood charcoal from the great fire of Berlin in A.D. 1980, found with excavation of the Nikolaikirche, Berlin-Mitte $\left(52^{\circ} 31^{\prime} \mathrm{N}\right.$ Lat, $13^{\circ} 24^{\prime} \mathrm{E}$ Long), Germany. Excavated 1957, coll. and subm. by E. Reinbacher, Institut für Vor- und Frühgeschichte der Deutschen Akademie der Wissenschaften zu Berlin (Reinbacher, 1963). Comment: historical age A.D. 1380 was confirmed by a coin from between A.D. 1370 and 1380, found within charred remains from fire (A. Suhle; see Reinbacher, 1963, p. 52).

\section{INTER-LABORATORY CHECKS}

\section{KN-10b. Langenlois}

$$
\begin{aligned}
& 26,560 \pm 1600 \\
& 24,610 \text { в.C. } \\
& 26,960 \pm 1200 \\
& 25,010 \text { в.c. }
\end{aligned}
$$

KN-10c. Langenlois

Wood charcoal from Hearth no. X/1961 of a Gravettian encampment found in loess at Langenlois (48 $28^{\prime} \mathrm{N}$ Lat, $15^{\circ} 40^{\prime} \mathrm{E}$ Long), near Krems, Niederösterreich, Austria. Excavated 1961 by F. Felgenhauer, Institut für Ur- und Frühgeschichte, Univ. of Vienna, Austria (see Felgenhauer, 1963). Coll. and subm. 1962 by F. Felgenhauer; final publication in preparation. Comment: material of same sample has been dated by Heidelberg lab.: H-2218/1537 (Sch-181), 25,480 \pm 880 в.P. (K. O. Münnich, pers, commun.).

\section{KN-51. Coveta del Or}

$6510 \pm 160$

4560 B.c.

Charred cereal grains (Triticum monococcum, det. Mrs. M. Hopf, Mainz) from supply-like find in a layer containing Cardial ceramics, in Coveta del Or cave, close to Benniarres near Alcoy, prov. Alicante $\left(38^{\circ}\right.$ $42^{\prime} \mathrm{N}$ Lat, $0^{\circ} 28^{\prime} \mathrm{W}$ Long), Spain. Excavated by V. P. Perez, coll. and subm. by H. Schubart, Deutsches Archäologisches Institut, Madrid (Schubart and Perez, 1964; Hopf and Schubart, 1965). Comment: sample from same place was dated at Heidelberg: H-1754/1208 (Sch-179), $6265 \pm 75$ B.P. (K. O. Münnich, pers, commun.).

\section{KN-21. Egolzwil 4}

$5370 \pm 160$

Wood (Abies) from small tree found with 1954 excavations of Egolzwil 4, Luzern $\left(47^{\circ} 11^{\prime} \mathrm{N}\right.$ Lat, $8^{\circ} 01^{\prime} \mathrm{E}$ Long), Switzerland, by E. Vogt, Zürich. Site belongs to Younger Cortaillod culture, lower layer. Tree found in horizonal position in chalky bottom layers of lake. Comment: wood of same sample dated at Heidelberg: H-228/276 Egolzwil 4 (Sch-66), $5150 \pm 100$. Another sample from same site, a board of ash (Fraxinus) wood found in upper layer of Younger Cortaillod culture (part of wooden floor of a house), was dated, too, at Heidelberg lab.: H-227/277 Egolzwil 4 (Sch-65), $5040 \pm 100$ B.P. (K. O. Münnich, pers. commum.). A date of Egolzwil 3 (belonging to Older Cortaillod cul- 
ture, see Vogt, 1955) was published by Copenhagen lab. (Copenhagen III, recalculated value; solid carbon technique measurements): K-115, $116,118,121$ (average), $4890 \pm 90$ в.P. Another date quoted by Zeuner (1958, p. 344) is F-17 Egolzwil 3, $4650 \pm 110$ B.P.

\section{KN-27. Riedschachen 13}

$5310 \pm 160$

Wood of oak beam from below a clay floor layer (threshold beam) of a house of Schussenried culture, find place Riedschachen 13 near Aichbühl/Federsee, Baden-Württemberg ( $48^{\circ} 02^{\prime}$ N Lat, $9^{\circ} 40^{\prime} \mathrm{E}$ Long), Germany. Sample taken 1963 by H. Schwabedissen, together with R. Schütrumpf and $\mathrm{E}$. Wall, upon excavating a test trench near border of earlier excavations by H. Reinerth (e.g., Zimmermann, 1961). Comment: another part from exactly the same piece of wood was taken during a trial excavation in 1962 by E. Wall and K. Göttlich (Göttlich, 1964), and submitted to Hannover lab. (see Hannover III): Hv-354, $5160 \pm 110$ B.P. Beam parts confirmed by E. Wall who collaborated with both excavations and also knows the older Reinerth excavations. Another sample dated at Hannover was Hv-353, $5130 \pm 100$.

General comment: date is comparable to that of Ehrenstein (KN-2, 191, this date list); a palynological study of both sites will be published by R. Schütrumpf.

\section{KN-2. Ehrenstein, Sch-170}

$5210 \pm 120$ 3260 B.c.

KN-191. Ehrenstein, 41b

Two samples, charred cereal grains (Sch-170) and wood charcoal (4lb), from Neolithic village Ehrenstein, Kreis Ulm, Baden-Württemberg ( $48^{\circ} 25^{\prime} \mathrm{N}$ Lat, $9^{\circ} 55^{\prime} \mathrm{E}$ Long), Germany. Village excavated in 1952 by O. Paret (Paret, 1955) and in 1960 by H. Zürn, Staatliches Amt für Denkmalpflege, Abt. Bodendenkmalpflege, Stuttgart (Zürn, 1962). Site belongs to Schussenried culture, with influence of Michelsberg culture. Samples taken during excavation campaign of 1960 by H. Schwabedissen and R. Schütrumpf, from Area no. 8, lane bewteen Houses 5 and 6 , depth $1.52 \mathrm{~m}$. Comment: material of same sample Sch-170 was also dated at Berlin and Heidelberg labs.: H-1749/1201, $5030 \pm 80$ B.P., and Bln-167, $5195 \pm 100$ B.P. (K. O. Münnich and H. Quitta, pers. commun.).

The following dates of comparable samples from same site were published (Berlin I): Bln-54, $5140 \pm 80$ B.P.; Bln-71, $5200 \pm 100$ B.P.; Bln-70, $5240 \pm 100$ B.P.; and also two samples from 1952 excavation (Heidelberg I; see also Groschopf, 1957): H-125/107, $5200 \pm 200$ B.P. and $\mathrm{H}-61 / 149,5140 \pm 130$ B.P.

Special comment (H.S.): the exact location of the 1952 samples has been questioned. 


\section{KN-38. Baia-Hamangia}

$4060 \pm 160$

2110 B.C.

Charcoal from Ochre Grave culture layer, Grave no. 1, in Burial Mound no. 1, at Baia-Hamangia, r. Istria, reg. Constanta $\left(44^{\circ} 47^{\prime} \mathrm{N}\right.$ Lat, $28^{\circ} 42^{\prime}$ E Long), Romania. Excavated 1952 and subm. by D. Berciu, Romanian Acad. of Sci., Archaeol. Inst., Bucharest, Romania, on commission of H. Quitta, Deutsche Akademie der Wissenschaften zu Berlin, Institut für Vor- und Frühgeschichte, as cross-check sample. Comment: material of same sample dated at Berlin lab. (Berlin I, Kohl and Quitta, 1963): Bln-29, Baia-Hamangia, $4090 \pm 160$ B.P. Sample from same grave was dated at Groningen lab. to give $2331 \pm 65$ B.C. (Berciu, 1961, p. 124), corresponding to the value Gro-1995, $4280 \pm 65$ B.P., as quoted by Kohl and Quitta (1963). Proper correction for the Suess effect as given in Groningen IV results in date there given as GrN-1995, Hamangia-2, $4530 \pm 65$ B.P.

\section{KN-8. Carmona}

$$
\begin{aligned}
& \mathbf{2 4 7 0} \pm 120 \\
& \mathbf{5 2 0} \text { B.C. }
\end{aligned}
$$

Wood charcoal from large charred area found during excavations of ancient town of Carmona, prov. Sevilla $\left(37^{\circ} 29^{\prime} \mathrm{N}\right.$ Lat, $5^{\circ} 37^{\prime} \mathrm{W}$ Long), Spain. This charred layer, no. 3, is attributed to a great fire, supposedly in third or fourth century B.c. (Raddatz and Carriazo, 1961, p. 104). Coll. and subm. 1959 by K. Raddatz, Institut für Ur- und Frühgeschichte der Universität Göttingen. Comment: material from same sample dated by Heidelberg lab.: H-1037/1550 (Sch-155), $2400 \pm$

\begin{tabular}{|c|c|}
\hline \multirow{2}{*}{\multicolumn{2}{|c|}{ REFERENCES }} \\
\hline & \\
\hline Berlin I & Kohl and Quitta, 1964 \\
\hline Copenhagen III & Tauber, 1960 \\
\hline Groningen IV & Vogel and Waterbolk, 1963 \\
\hline Hannover III & Geyh and Schneekloth, 1964 \\
\hline Heidelberg I & Münnich, 1957 \\
\hline USGS I & Suess, 1954 \\
\hline USGS II & Rubin and Suess, 1955 \\
\hline USGS III & Rubin and Suess, 1956 \\
\hline Yale III & Barendsen, Deevey, and Gralenski, 1957 \\
\hline
\end{tabular}
50 в.P. (K. O. Münnich, pers. commun.).

Albrecht, Chr., 1938/1942, Das Römerlager in Oberaden, 2 vols.: Dortmund, Ruhfus.

Almagro, M., and Arribas, A., 1963, El poblado y la necropolis megalithicos de Los Millares: Madrid, Bibliotheca Praehistorica Hispana.

Anderson, E. C., Libby, W. F., Weinhouse, S., Reid, A. F., Kirshenbaum, A. D., and Grosse, A. V., 1947, Radiocarbon from cosmic radiation: Phys. Rev., v. 72, p. 931-936.

Arnold, J. R., and Libby, W. F., 1949, Age determination by radiocarbon content. Checks with samples of known age: Science, v. 110, p. 678-680.

Aschemeyer, H., 1963, Neue Untersuchungen im Römerlager Oberaden: Prähistorische Zeitschrift, v. 41, p. 210-212.

Barendsen, G. W., Deevey, E. S., and Gralenski, L. J., 1957, Yale natural radiocarbon measurements III: Science, v. 126, p. 908-919.

Beckhoff, K., 1960, Grundlagen und Beschreibung einer einfachen Elektronik für die Messung von C-14 mittels eines $\mathrm{CO}_{2}$-Proportionalzählrohrs: ms., Witten.

Berciu, D., 1961, Contributii la problemele neoliticului in Romania in lumina noilor cercetari: Bucuresti, Editura Academiei. 
Bittel, K., Naumann, R., Beran, Th., Hachmann, R., and Kurth, G., 1957, Bogazköy III: Berlin, Mann.

Calvin, M., Heidelberger, C., Reid, J. C., Tolbert, B. M., Yankwich, P. F., 1960, Isotopic carbon: New York, Wiley.

Felgenhauer, F., 1962/1963, Ein geschäfteter Klingenkratzer aus dem niederösterreichischen Jungpaläolithikum: Acta Archacologica (Ljubljana, Slovenic Academy), v. $13 / 14$, p. $61-69$.

Firbas, F., 1953, Das absolute Alter der jüngsten vulkanischen Eruptionen im Bereich des Laacher Sees: Naturwissenschaften, v. 40, p. 54-55.

Freundlich, J., 1965, A radon effect with a copper counting tube: ms., Internat. C-14 Dating Conf.

Geyh, M. A., and Schneekloth, H., 1964, Hannover Radiocarbon measurements III; Radiocarbon, v. 6, p. 251-258.

Göttlich, K. H., 1963/1964, Die ersten Radiocarbondaten einiger Siedlungsschichten im Federseemoor: Jahresh. Ver. Vaterländ. Naturkunde Württemberg (Stuttgart), v. $118 / 119$, p. $208-210$.

Groschopf, P., 1957, Das Alter des Steinzeitdorfes Ehrenstein Kr. Ulm nach C-14Bestimmungen: Fundber. aus Schwaben (Stuttgart), v. 14, p. 132.

Gross, H., 1952, Die Radiocarbonmethode, ihre Ergebnisse und Bedeutung für die spätquartäre Geologie, Paläontologic und Vorgeschichte: Eiszeitalter u. Gegenwart, v. 2, p. $68-92$.

1954, Das Alleröd-Interstadial als Leithorizont der letzten Vereisung in Europa und Amerika: Eiszeitalter u. Gegenwart, v. 4/5, p. 189-209.

1955, Weitere Beiträge zur Kenntnis des Spätglazials: Eiszeitalter $u$. Gegenwart, v. 6, p. 110-115.

Harteck, P., 1951, Chemie und Photochemie in der Erdatmosphäre: Angewandte Chemie, v. 63, p. 1-7.

Haxel, O., 1957, Geologische und archäologische Datierungen mit C-14: Naturwissenschaften, v. 44, p. 163-169.

Hopf, M., and Schubart, H., 1965, Neue Daten für cardialkeramische Schichten der Coveta del Or: ms., Madrid and Mainz.

Kohl, G., and Quitta, H., 1963, Berlin-Radiocarbondaten archäologischer Proben, I: Ausgrabungen und Funde, v. 8, p. 281-301.

1964, Berlin radiocarbon measurements I: Radiocarbon, v. 6, 308-317.

Münnich, K. O., 1957, Heidelberg natural radiocarbon measurements I: Science, v. 126 p. $194-199$.

Paret, O., 1955, Das Steinzeitdorf Ehrenstein bei Ulm (Donau): Stuttgart, Schweizerbarth.

Petrikovits, H. von, 1957, Novaesium, das römische Neuss: Köln, Böhlau.

Raddatz, K., and Carriazo, J. de M., 1961, Ergebnisse einer ersten stratigraphischen Untersuchung in Carmona: Madrider Mitt., v. 2, p. 71-106.

Reinbacher, E., 1963, Die älteste Baugeschichte der Nikolaikirche in Alt-Berlin: Berlin, Akademieverlag.

Rubin, M., and Suess, H. E., 1955, U.S. Geological Survey radiocarbon dates II: Science, v. 121 , p. $481-488$.

1956, U.S. Geological Survey radiocarbon dates III: Science, v. 123, p. $442-448$

Sangmeister, E., and Do Paço, A., 1956, Vila Nova de S. Pedro, eine befestigte Siedlung der Kupferzeit in Portugal: Germania, v. 34, p. 211-230.

Schlabow, K., 1961, Der Moorleichenfund von Peiting: Neumünster, Wachholz.

Schubart, H., and Perez, V. P., 1964, Nuevas fechas del C-14 para los estratos de ceramica cardial en la Coveta del Or: Archivo de Prehistoria Levantina, v. 11, in press.

Schwabedissen, H., 1949, Die Bedeutung der Moorarchäologie für die Urgeschichtsforschung: Offa-Zeitschr. (Neumünster, Wachholz), v. 8, p. 46-74.

Schwabedissen, H., and Münnich, K. O., 1958, Zur Anwendung der C-14-Datierung und anderer naturwissenschaftlicher Hilfsmittel in der Ur- und Frühgeschichtsforschung: Germania, v. 36, p. 133-149.

Suess, H. E., 1954, U.S. Geological Survey radiocarbon dates I: Science, v. 120, p. 467-473.

Tauber, H., 1960, Copenhagen natural radiocarbon measurements III: corrections to radiocarbon dates made with the solid carbon technique: Am. Jour. Sci. Radioc. Supp., v. 2, p. 5-11. 
Vogel, J. C., and Waterbolk, H. T., 1963, Groningen radiocarbon dates IV: Radiocarbon, v. 5, p. 163-202.

Vogt, E., 1955, Pfahlbaustudien: in Das Pfahlbauproblem, W. U. Guyan (ed.) : Basel, Birkhäuser, p. 199-222.

Vries Hl. de, and Barendsen, G. W., 1953, Radiocarbon dating by a proportional counter filled with carbon dioxide: Physica, v. 19, p. 987-1003.

Zeuner, F. E., 1951, Archäologische Zeitbestimmung durch radioaktiven Kohlenstoff: Archaeologica Austriaca, v. 8, p. 82-94.

1958, Dating the Past, 4th ed.: London, Methuen.

Zimmermann, W., 1961, Der Federsee: Stuttgart, Schwäb.Alb-Verein.

Zoller, D., 1963, Das Kreisgraben-Gräberfeld Querenstede, Kr. Ammerland: Die Kunde (Hannover), v. 14, p. 98-111.

Zürn, H., 1962, Neue Ausgrabungen im Jungsteinzeitdorf bei Ehrenstein, Kr. Ulm (Donau): Germania, v. 40, p. 126-130. 